\title{
Early spring ephemeral therophytic non-nitrophilous grasslands as a habitat of various species of Romulea in the southern Balkans
}

\author{
ANDRAŽ ČARnI $^{1 *}$, Vlado MATEVSKI $^{2}$, URBAN ŠILC ${ }^{1}$, ReNATA ĆUŠTEREVSKA $^{2}$ \\ ${ }^{1}$ Institute of Biology, Scientific Research Center of the Slovenian Academy of Sciences \\ and Arts, Novi trg 2, SI 1000 Ljubljana, Slovenia \\ ${ }^{2}$ Institute of Biology, Faculty of Natural Sciences of St. Cyril and Methodius University \\ in Skopje, Gazi baba b/b, MK 1000 Skopje, Republic of Macedonia
}

\begin{abstract}
The work deals with habitats of Romulea bulbocodium and Romulea linaresii ssp. graeca in the southern Balkans. Both species appear in early spring ephemeral therophytic non-nitrophilous grasslands in regions under the influence of the Mediterranean climate. These communities are classified within the Romulion alliance, which encompasses such communities from the eastern Mediterranean area. It was established that the main climatic factor causing the diversity of these communities is seasonality in precipitation and temperature. Two associations are presented, as Lagopo-Poetum bulbosae and Romuleo graecae-Poetum bulbosae.
\end{abstract}

Key words: Balkans, climate, grassland, nomenclature, Romulea, vegetation

Abbreviations: ESETG - early spring ephemeral therophytic non-nitrophilous grasslands, ICPN - International Code of Phytosociological Nomenclature

\section{Introduction}

In the early spring, from the end of February to the end of March, carpets of flowering plants belonging to the genus Romulea appear in areas under the influence of the Mediterranean climate (Fig. 1). Later, at the end of April and beginning of May, when these species-rich communities are optimally developed for sampling, Romulea species are already in fruit and can no longer be identified. In summer, early spring ephemeral therophytic non-nitrophilous grasslands (ESETG) dry out due to the hot and dry Mediterranean climate. Only a few drought-resistant species can then be found, e.g., Achnatherum bromoides, due to $\mathrm{C} 4$ assimilation syndrome (PYANKOv et al. 2010), and only green-grey remains of the colorful spring carpet.

* Corresponding author, e-mail: carni@zrc-sazu.si

Copyright ${ }^{\circledR} 2014$ by Acta Botanica Croatica, the Faculty of Science, University of Zagreb. All rights reserved. 


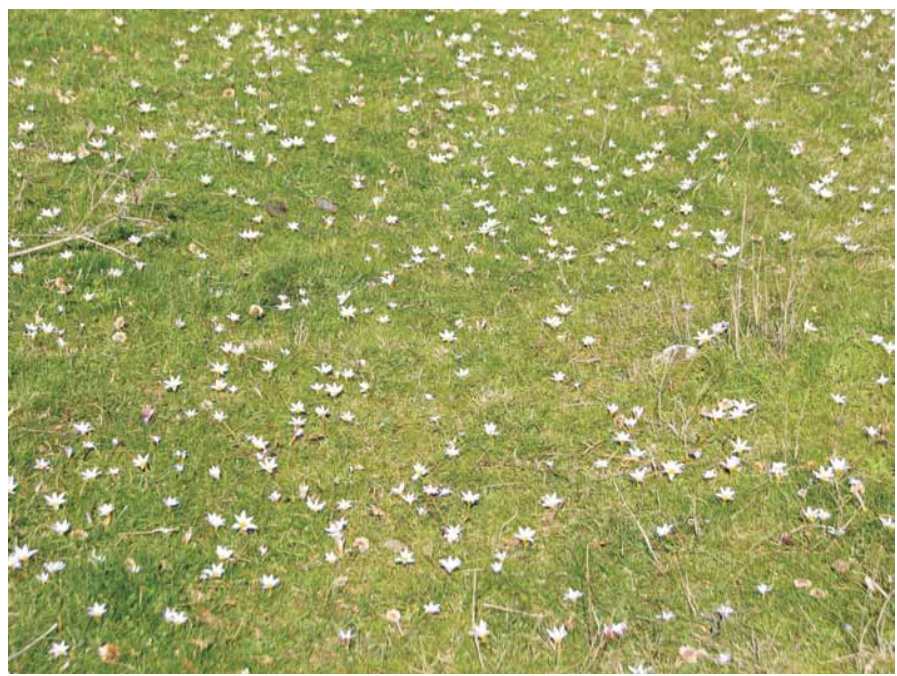

Fig. 1. View of ESETG in the end of March. Romulea in full flower. At the time of sampling in the beginning of May up to 100 plant species can be found in a single plot.

The genus Romulea comprises about 95 taxa, of which 80 occur in South Africa and the Arabian peninsula (MANNING and GoldBlatT 2008), while the others can be found in the Mediterranean basin (PERUZZI et al. 2011). Our study deals with the habitats of two species: Romulea bulbocodium, which is widespread in the Mediterranean basin, and Romulea linaresii ssp. graeca, an endemic species of the Balkan Peninsula (FRIGNANI and IIRITI 2011) (Fig. 2). Two other subspecies of $R$. linaresii, subsp. linaresii and abyssinica, are endemic to Sicily and Ethiopia, respectively (PIGNATTI 1982).

Romulea bulbocodium is a quite tall plant up to $15 \mathrm{~cm}$ and has $1-6$ flowers that are lilac or violet, often greenish outside with a yellow throat and tube (Fig. 2A). It has a wider distribution in the Balkans than $R$. linaresii, being found along the Adriatic coast, in Macedonia, Bulgaria, Greece and Turkey (Nikolić 2000, SteŠEVIĆ 2002, Gussev 2011, NATChEVA and IVANOVA 2011).
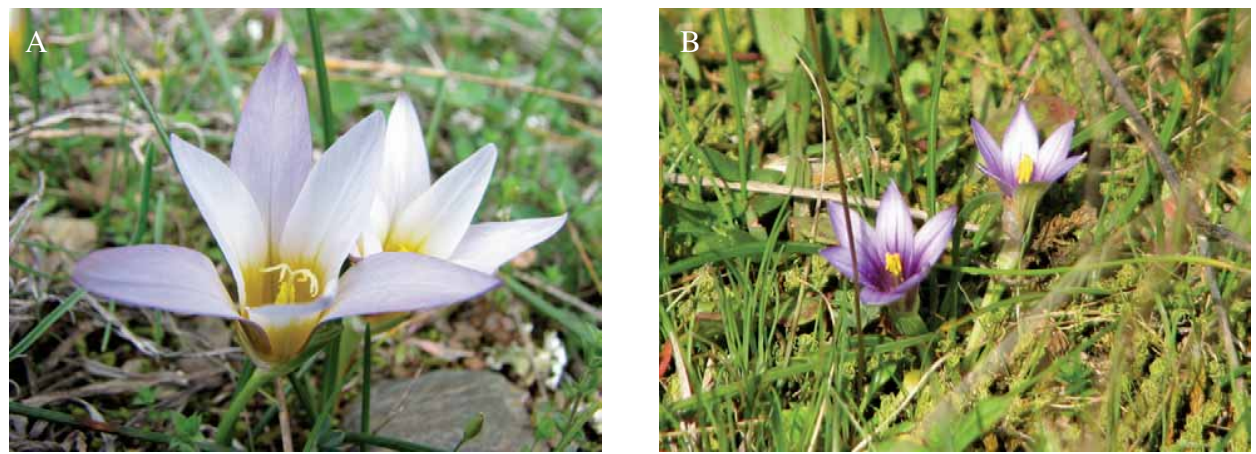

Fig. 2. Romulea bulbocodium (A) is found in the interior part of the region, whereas Romulea linaresii ssp. graeca $(\mathrm{B})$ appears in the coastal part. 
Romulea linaresii ssp. graeca is smaller than $R$. bulbocodium. It can be up to $5 \mathrm{~cm}$ tall with 1-2 dark violet-purple flowers (Fig. 2B) and is distributed only in the southern part of the Balkans and Turkey (e.g. STEŠEvić 2002, ÖZdemir et al. 2007, Petrova and Vladimirov 2009, BERGMEIER et al. 2011, GuSSEV 2011, RAKAJ 2011).

There is lively interest in the taxonomical peculiarities and distribution of Romulea species in the Balkans but there have been only a few investigations dealing with the habitats of these species: ESETG (OBERDORFER 1954, Bolòs et al. 1996). Sampling of these communities is rather difficult due to high biological and phenological diversity.

Grasslands are species-rich communities established over centuries of permanent grazing (KALIGARIČ et al. 2006, CATORCI et al. 2012). As well as by grazing, grasslands in the Mediterranean area are also maintained by periodic fires (KAVGACI et al. 2010, TÜRKMEN and DüNZENLI 2011). In recent years, due to land use change (BRACCHETTI et al. 2012), grasslands have become among the most threatened habitats (JANIŠOVÁ et al. 2011, VASSILEV et al. 2011), since the communities often become overgrown (BARBERO and QUEZEL 1976, ČARNI et al. 2010). OBERDORFER (1954) sampled such habitats in 1944 in the areas around Thessaloniki, southern Macedonia, Thrace, Thessaly, Attica and close to Corinth. He designated these communities that are mainly dominated by Poa bulbosa ssp. div. and which can be found on fresh, fine argillous soils around intensively grazed places. He described the alliance Romulion within this framework. He also stated that such vegetation is rarely found on limestone since Cisto-Micromerietea vegetation mainly appears there. BoLòs et al. (1996) later also elaborated such habitats on Cephalonia (Ionian islands, Greece).

In addition to ESETG found in areas under the influence of the Mediterranean climate, other types of therophytic grasslands exist in more continental parts of the Balkans (e.g., in Bulgaria and the Republic of Macedonia). These communities are classified within Trifolion cherleri. The classification of the latter alliance into higher rank syntaxa is still under consideration, since they mediate between the group of (sub-) Mediterranean therophytic grasslands (Helianthemetea) and more continentally influenced perennial grasslands (Festuco-Brometea) (MicEVski 1970, TzONEV et al. 2009). There are even discrepancies at the association level, for instance within Erysimo-Trifolietum (MicEVsKi 1977, SOPOTLIEVA and Apostolova 2007).

There is a well recognizable climatic gradient in the southern Balkans: Mediterranean sub-Mediterranean - continental, which provides the opportunity to study in detail the turn-over of plant species and communities under changing climatic conditions (ĆUŠTEREVSKA et al. 2012).

RoDwELL et al. (2002) defined the alliance Romulion as eastern Mediterranean ephemeral vegetation on humid salty soils. They place this alliance within Saginetea maritimae, a class of ephemeral vegetation with winter annuals on bare or disturbed salt-marsh mud and sand.

The aim of the study was to sample ESETG in the southern Balkans and define the habitats of Romulea species in the region. We have tried to present in the paper the floristic composition of communities and to analyze their structural and choraological spectrum. We have determined the most important climatic factor for diversity within communities and correlated it with their geographical distribution. We have attempted to discover the ecological and syntaxonomic position of ESETG and have performed a nomenclatural revision of these syntaxa. 


\section{Material and methods}

The research area was the southern part of the Balkans (Fig. 3). According to the Map of Natural Vegetation of Europe (BOHN et al. 2003), the northern part of the area in which Romulea species appear lies in the zone of thermophilous deciduous broadleaved forests (Carpinus orientalis-Quercus pubescens zone), whereas the southern part along the Aegean coast lies in the zone of Mediterranean evergreen forests dominated by Quercus ilex. The northern limit of growth of Romulea species corresponds to the northern boundary of thermophilous deciduous broadleaved forests as defined by HORVAT et al. (1974) and the distribution of Quercus coccifera scrub or forest (OBERDORFER 1947).

The climatological station in Valandovo (southern part of the Republic of Macedonia) reports that there is $611 \mathrm{~mm}$ mean annual precipitation and the mean annual temperature is $14.6{ }^{\circ} \mathrm{C}$; in Thessaloniki, on the coast, there is $458 \mathrm{~mm}$ of mean annual precipitation and a mean annual temperature of $15.8^{\circ} \mathrm{C}$. In both stations, the highest peak of precipitation is in November with a less pronounced peak in May in Valandovo and in March and May in Thessaloniki (MATEVSKI and ČARNI 2003).

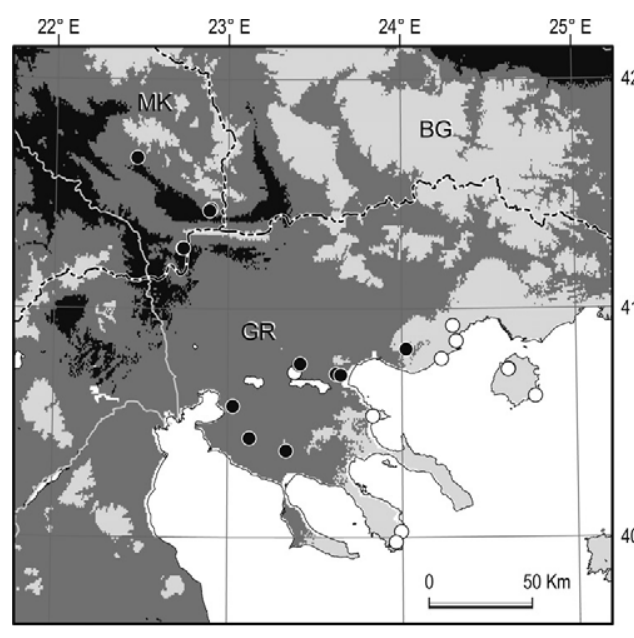

BIO 4 - Temperature Seasonality

$5684-6910$

$6911-7550$

$7551-7892$

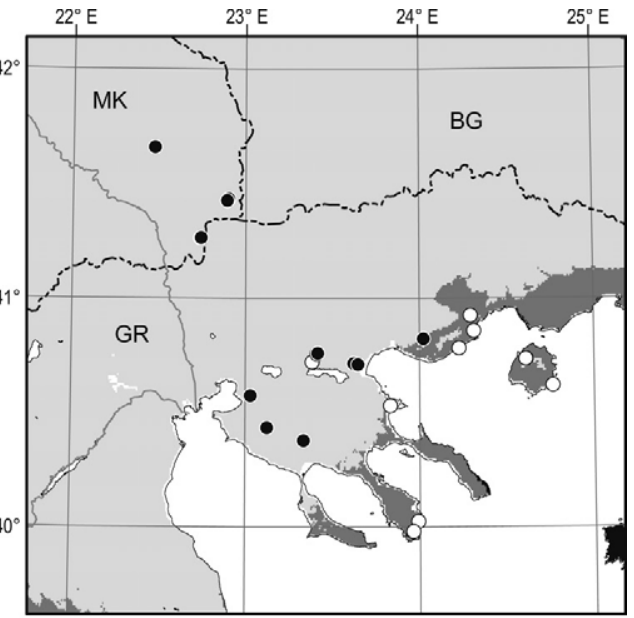

BIO 15 - Precipitation Seasonality

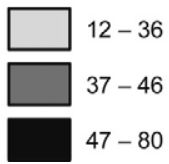

Fig. 3. Distribution of communities: communities with Romulea bulbocodium are distributed in the inland part and represented by full circles; communities with Romulea linaresii ssp. graeca are distributed in coastal areas and represented by empty circles. In the map two layers generated through interpolation of climate data stored in WorldClim database are presented. The numbers in BIO 4 present the coefficient of variation as standard deviation of the weekly mean temperatures expressed as a percentage of the annual mean, in this case multiplied by 100 , whereas BIO 15 also presents the coefficient of variation as previously, although in this case precipitation is multiplied by 10 (HIJMANS et al. 2005). 
Sampling was performed according to the standard Braun-Blanquet method (BRAUNBLANQUET 1964). The layer is not indicated, because all species appear in the herb layer. Since it is practically impossible to determine Romulea species when they are fruiting, we visited the sites three times. In early spring, we surveyed the whole area, identified potential plots for sampling communities and noted geophytes, such as Romulea, Gagea, Ornithogalum etc. At the end of April and beginning of May, we performed field sampling of vegetation. At this time, we made an inventory of plants and estimated coverage. We visited plots once more in summer, in order to find plants, above all grasses, which had not been in flower at sampling time. These species mainly possess a $\mathrm{C} 4$ photosynthesis pathway and do well under high light intensity and high temperatures. They include Achnatherum, Bothriochloa, Chysopogon, and also the non-grass Portulaca.

After elaboration of the plant material, we constructed a table (Tab. 1), which was subjected to numerical analysis. Classification was then carried out by PC-ORD (MCCUNE and MEFFORD 2011), run in the JUICE 7.0 programme (TiCHÝ 2002). A dendrogram was drawn using Ward's method and Euclidean distance as a measure of resemblance (Fig. 4).

The characteristic species of individual groups were determined by calculating the species' fidelity and they are presented in the analytic table (Tab. 1). The phi coefficient was used as a fidelity measure and calculated in the JUICE program. The threshold phi value (multiplied by 100 in the JUICE program) for species to be considered characteristic was set at 40 (CHYTRÝ et al. 2002).

All the climatic data available in the WorldClim database (HIJMANS et al. 2005, available at http://worldclim.org/) were extracted for each sample plot. Before elaboration, we firstly performed detrended correspondence analysis (DCA) of data and discovered that the gradient is less than $3 \mathrm{SD}$, indicating linearity, and we therefore used prinicipal component analysis (PCA) and redundancy analysis (RDA) in further analysis. Since climatic data were provided from an external source and not from a species inventory of ESETG (such as life forms and chorotypes), RDA was used to test the possible effect of climatic variables on vegetation composition. Each variable was entered separately, each in turn, for analysis and

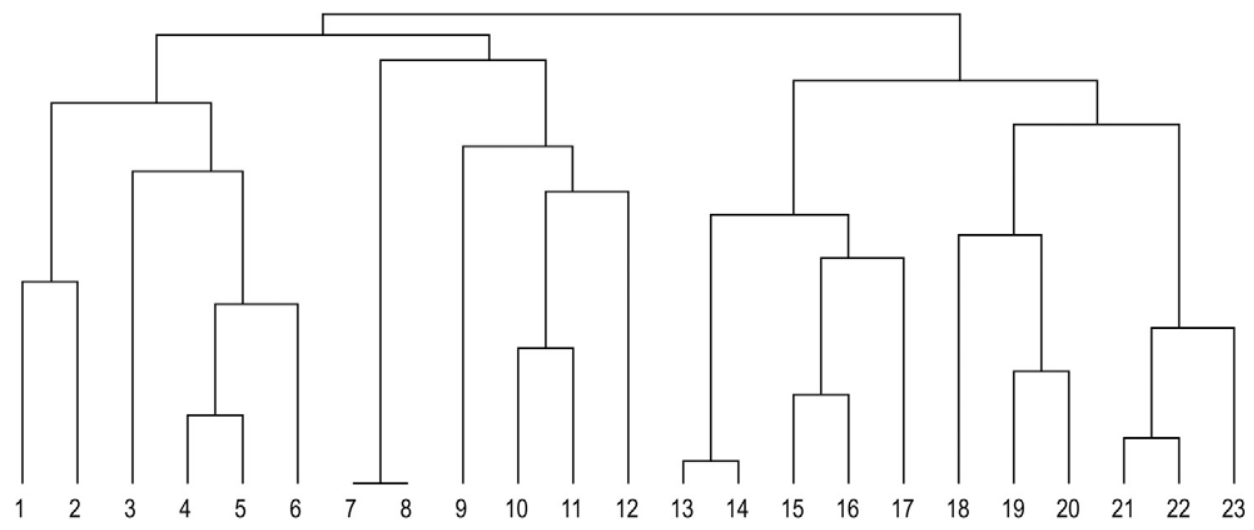

Fig. 4. Dendrogram of samples (relevés). Two main clusters can be seen, inland communities with Romulea bulbocodium are indicated by number 1-12, whereas the coastal communities with Romulea linaresii ssp. graeca are indicated by number 13-23. 
Tab. 1. Analytical table of the early spring ephemeral therophytic non-nitrophilous grasslands in the Southern Balkan.

\begin{tabular}{lll|lllllllllll}
1 & 1 & 1 & 1 & 1 & 1 & 1 & 1 & 1 & 1 & 2 & 2 & 2 & 2
\end{tabular}

Relevé number

\begin{tabular}{llllllllllll|lllllllllll}
1 & 2 & 3 & 4 & 5 & 6 & 7 & 8 & 9 & 0 & 1 & 2 & 3 & 4 & 5 & 6 & 7 & 8 & 9 & 0 & 1 & 2 & 3
\end{tabular}

\section{LAGOPO-POETUM BULBOSAE}

Romulea bulbocodium

Myosotis ramosissima

Teucrium capitatum

Alyssum desertorum

Achillea coarctata

Carex caryophyllea

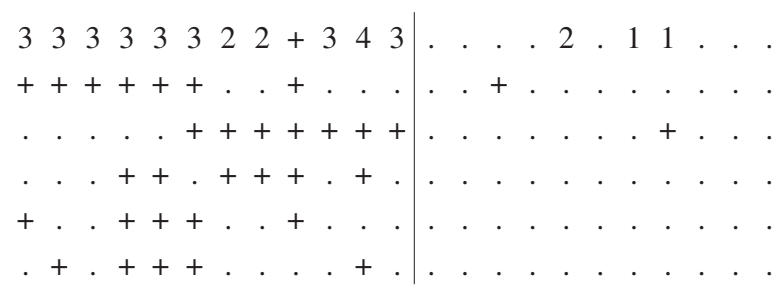

\section{ROMULEO GRAECAE-POETUM BULBOSAE}

Romulea linaresii ssp. graeca

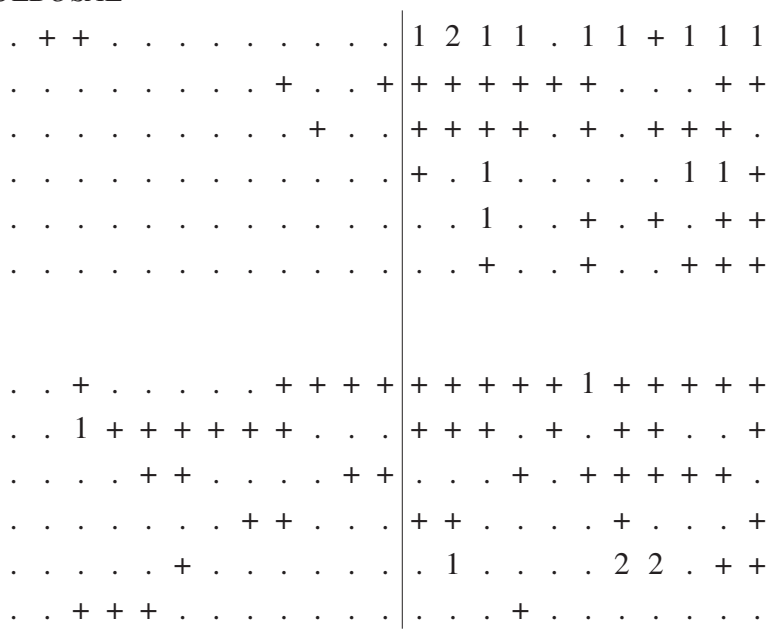

Galium murale

Hordeum murinum ssp. leporinum

Lagurus ovatus

Crepis zacintha

Urospermum picroides

\section{ROMULION}

Hedypnois rhagadioloides

Hypochoeris cretensis

Allium guttatum

Linaria simplex

Ornithogalum collinum

Lotus angustissimus

\section{HELATHEMETALIA,HELIATHEMETEA}

\section{Poa bulbosa}

Psilurus incurvus

Vulpia ciliata

Tuberaria guttata

Trifolium campestre

Ornithopus compressus

Trifolium angustifolium

Hypochaeris glabra

Trifolium arvense

Plantago bellardii

Linaria pelisseriana

Trifolium subterraneum

Trifolium cherleri \begin{tabular}{lllllllllllll|lllllllllll}
3 & 2 & 3 & 2 & 3 & 2 & 4 & 4 & 4 & 3 & 3 & 2 & 4 & 3 & 3 & 3 & 3 & 4 & 3 & 2 & 2 & 3 & 2
\end{tabular}

. $++++1++$ + $+2++2+++++++++$

. $+1++++++. .++.11++1+31+$

$.12+32+\ldots .++1+22211+212$

$+.122+. .++.+++1 .+21+++1$

$+.11+.++$. . . + $1++2++++.++$

$+.++++.++1.2 .+++.1+++1+$

. $1++++++$. . . ++ . ..+++++++

. . $+11+++.+.1$. . . $++++++1+$

.. $1+2+. .++2 \cdot 1+21++1121$

. . +++++ . . . . . . $+++1++++++$

$322++.+$. . . . $2++2+. .+.1+$

. . 1 . $12+$. . . 1 . . $+++324++2$ 
Tab. 1. - continued

\begin{tabular}{lll|lllllllllll}
1 & 1 & 1 & 1 & 1 & 1 & 1 & 1 & 1 & 1 & 2 & 2 & 2 & 2
\end{tabular}

Relevé number $\begin{array}{lllllllllllllllllllllll}1 & 2 & 3 & 4 & 5 & 6 & 7 & 8 & 9 & 0 & 1 & 2 & 3 & 4 & 5 & 6 & 7 & 8 & 9 & 0 & 1 & 2 & 3\end{array}$

Aira elegantissima

Asterolinon linum-stellatum

Galium divaricatum

Medicago minima

Arenaria leptoclados

Filago gallica

Trifolium stellatum

Briza maxima

Tolpis umbellatum

Helianthemum salicifolium

Velezia rigida

Taeniatherum caput-medusae

Sedum caespitosum

Trachynia distachya

Hymenocarpos circinnatus

Viola kitaibeliana

Arenaria serpyllifolia

Vulpia myuros

Rostraria cristata

Crucianella latifolia

Alkanna tinctoria

Medicago disciformis

var. disciformis

Helianthemum aegyptiacum

Petrorhagia prolifera

Medicago disciformis

var. strumensis

Clypeola jonthlaspi

Dasypyrum villosum

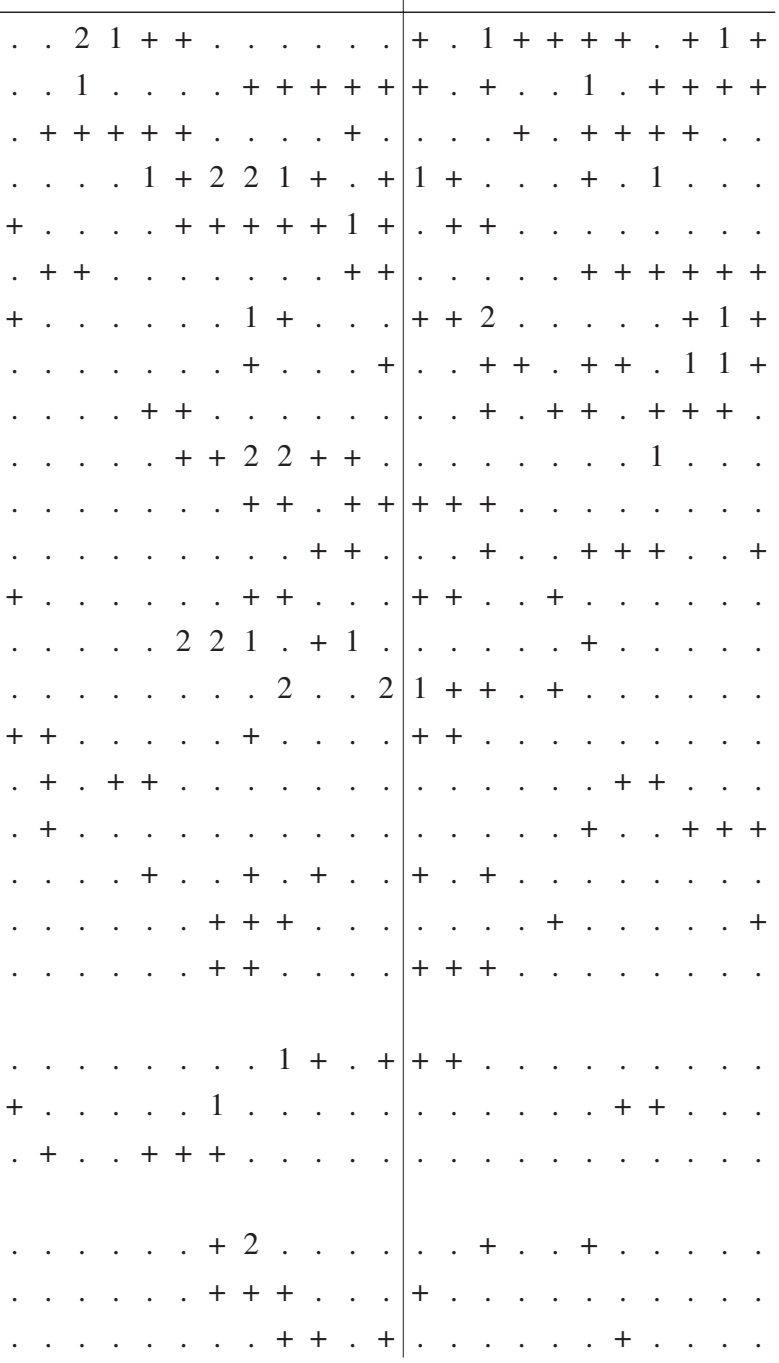

\section{FESTUCO-BROMETEA}

Trifolium scabrum

Eryngium campestre

Aegilops triuncialis

Chrysopogon gryllus

Medicago rigidula

Thymus substristus

Scleranthus verticillatus

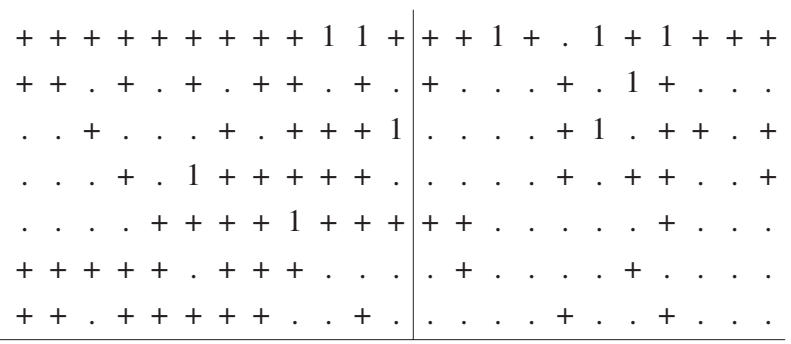


Tab. 1. - continued

Relevé number $\begin{array}{lllllllllllllllllllllll}1 & 2 & 3 & 4 & 5 & 6 & 7 & 8 & 9 & 0 & 1 & 2 & 3 & 4 & 5 & 6 & 7 & 8 & 9 & 0 & 1 & 2 & 3\end{array}$

Chondrilla juncea

Potentilla recta ssp. laciniosa

Bromus squarrosus

Arabidopsis thaliana

Centaurea grisebachii

Hypericum perforatum

Koeleria splendens

Achillea setacea

Sanguisorba minor

Convolvulus cantabrica

Astragalus onobrychis

Thymus striatus

Allium sphaerocephalon

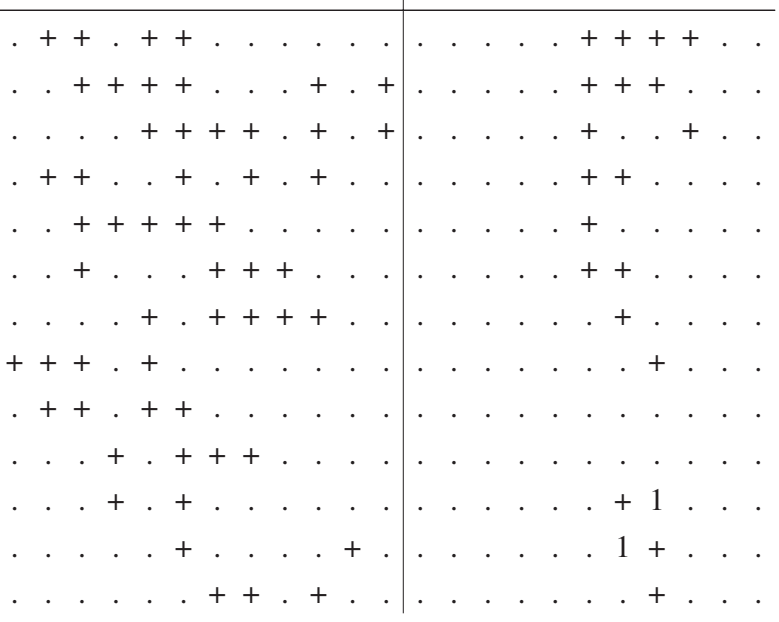

\section{OTHER SPECIES}

Cynodon dactylon

Parentucellia latifolia

Filago germanica

Anthemis auriculata

Petrorhagia velutina

Sherardia arvensis

Plantago lagopus

Erodium cicutarium

Achnatherum bromoides

Avena barbata

Veronica arvensis

Cistus incanus ssp. creticus

Trifolium retusum

Valerianella turgida

Senecio vulgaris

Trifolium nigrescens

Trifolium tenuifolium

Carthamus lanatus

Dactylis glomerata

Vicia lathyroides

Anagallis arvensis

Bothriochloa ischaemum

++++11 . . + . + $1 \mid++++1 .+++++$

$+111+1+++.+.++.2+.++.+1$

++++++ . +++++++++++ . .

+++ . . $11+1 .+1+11+.1+1+1$

..+++++ . . . $+1++++1++1+1$

$++1 .++. \cdot 1++++.++.1 .++++$

$+++.+.23+313+.1 .+12+3$

$++. .+++.++++1+++2 .++. .+$

$.+.+.++1+. \mid$ | . ...+++++++

. ..++ . +++++ . . . . +++++++

.+++ . . . . . . . $+.++1+$. . +++

. ++ . . . . ......++++++++++

. . +++ . . . . . . + . . $+1.1+++++$

.....++++++ . . . ...++++++

. . + . . . +++ . . .....+++++++

.++++ . . . . . .....+++++ . .

.+++ . + . . . . + . . + +. ..+ .1 .

++ . ..+++++ . . . . . . . . ..+++ .

......+++++ . . . . ..+++++

$+. .++.++. .++++. . \quad . \quad . \quad+.+$

. ...++++++++ . . . ...+ . . .

Polycarpon tetraphyllum

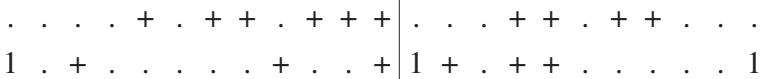


Tab. 1. - continued

\begin{tabular}{lll|lllllllllll}
1 & 1 & 1 & 1 & 1 & 1 & 1 & 1 & 1 & 1 & 2 & 2 & 2 & 2
\end{tabular}

Relevé number $\begin{array}{lllllllllllllllllllllll}1 & 2 & 3 & 4 & 5 & 6 & 7 & 8 & 9 & 0 & 1 & 2 & 3 & 4 & 5 & 6 & 7 & 8 & 9 & 0 & 1 & 2 & 3\end{array}$

Plantago lanceolata

Plantago coronopus

Cerastium semidecandrum

Moenchia erecta

Bromus hordeaceus

Aegilops geniculata

Onobrychis caput-galli

Asparagus acutifolius

Verbascum sinuatum

Veronica verna

Cerastium glomeratum

Portulaca oleracea

Eragrostis minor

Cynosurus echinatus

Crupina crupinastrum

Catapodium rigidum

Silene gallica

Crepis neglecta

Linum corymbulosum

Aphanes microcarpa

Rumex acetosella

Capsella bursa-pastoris

Crepis foetida ssp. rhoedifolia

Valerianella coronata

Echium plantagineum

Daucus guttatus

Astragalus pelecinus ssp. pelecinus

Centaurea benedicta

Holosteum umbellatum

Herniaria incana

Erysimum crassistylum

Lolium perenne

Anisantha tectorum

Crepis sancta

Medicago praecox

Anisantha madritensis

Ranunculus ficaria

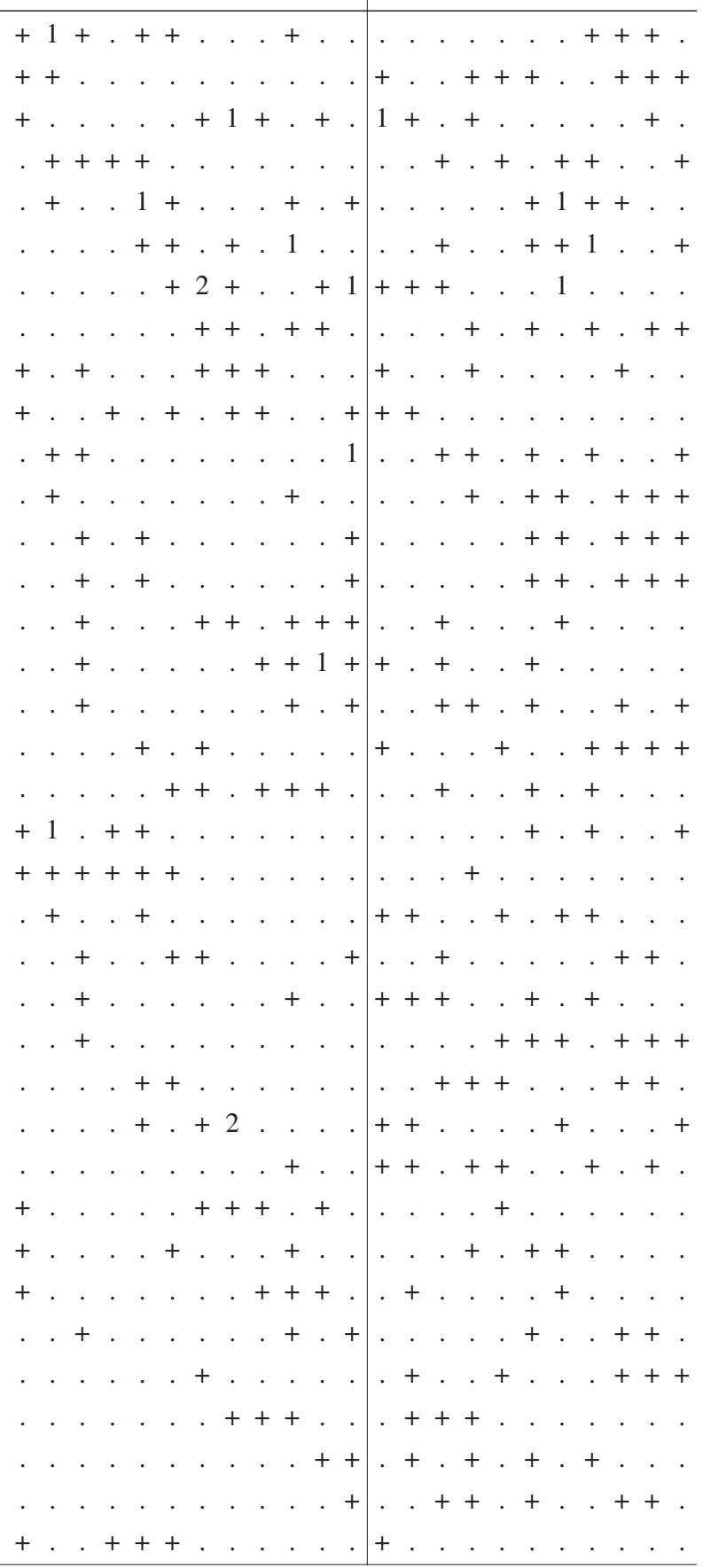


Tab. 1. - continued

$$
\begin{array}{lll|lllllllllll}
1 & 1 & 1 & 1 & 1 & 1 & 1 & 1 & 1 & 1 & 2 & 2 & 2 & 2
\end{array}
$$

Relevé number

\begin{tabular}{llllllllllll|lllllllllll}
1 & 2 & 3 & 4 & 5 & 6 & 7 & 8 & 9 & 0 & 1 & 2 & 3 & 4 & 5 & 6 & 7 & 8 & 9 & 0 & 1 & 2 & 3
\end{tabular}

Euphorbia helioscopia

Minuartia hirsuta ssp. falcata

Crepis setosa

Teesdalia coronopifolia

Vicia sativa ssp. nigra

Thymus heterotrichus

Anthoxanthum odoratum

Neatostema apulum

Allium flavum

Melica ciliata

Rorippa thracica

Geranium molle ssp. brutium

Filago arvensis

Teucrium chamaedrys

Nigella arvensis

Convolvulus elegantissimus

Sedum hispanicum

Anthemis ruthenica

Sedum rubens

Colchicum autumnale

Galium setaceum

Leopoldia comosa

Calicotome villosa

Centaurea orphanidea

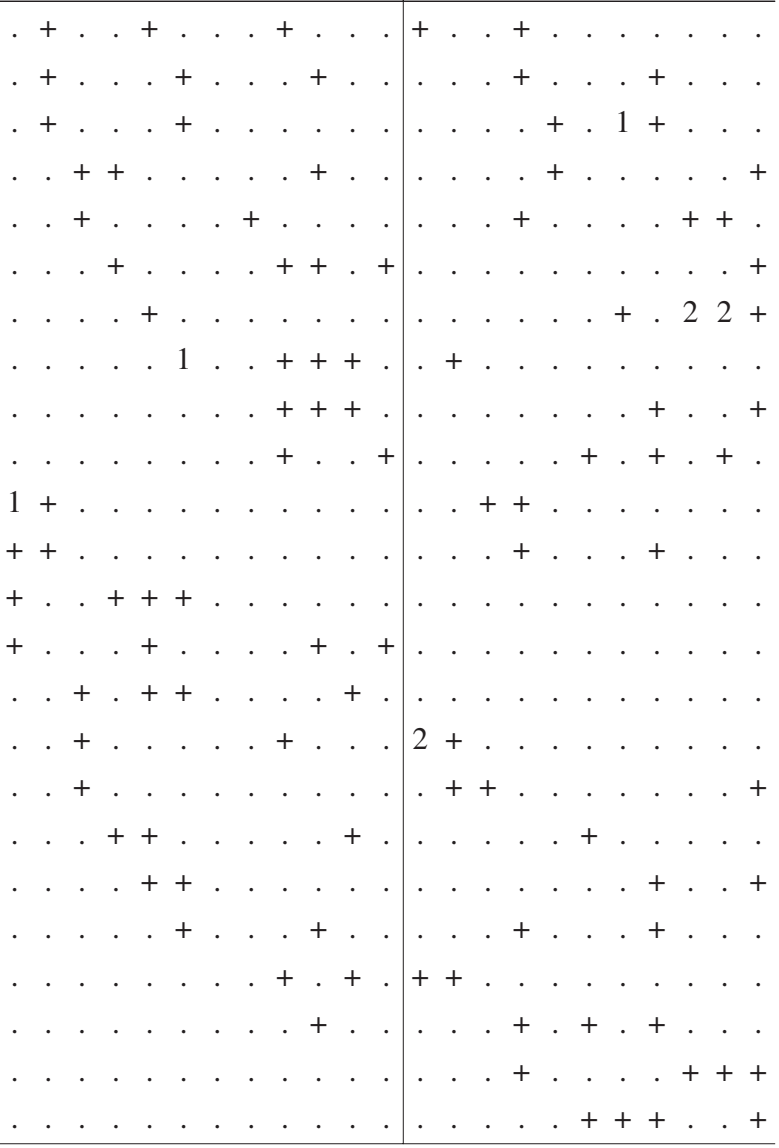

Other species: Agrostis stolonifera 14: +, 13: +; Alyssum corymbosoides 18: +; Aurinia corymbosa 16: +; Alyssum foliosum 5: +; Alyssum murale 18: 1, 10: +; Alyssum strigosum 19: +, 6: +; Alyssum umbellatum 8: +, 7: +; Amaranthus albus 14: +, 13: +, 16: +; Anemone pavonina 12: +; Apera spica-venti 16: +; Aphanes arvensis 3: +, 16: +; Asperula aristata ssp. scabra 3: +; Asphodeline lutea $2:+, 1: 1$; Asphodelus ramosus $\mathrm{ssp}$. ramosus $2:+, 1:+, 4:+;$ Astragalus spruneri $20:+;$ Bellis perennis 11: +, 14: +; Berteroa orbiculata 9: +; Anisatha sterilis 19: +, 10: +; Buglossoides arvensis 3: +; Bunias erucago 6: 1, 7: +; Bupleurum commutatum 23: +, 9: +; Calendula arvensis 5: +, 6: +, 13: +; Campanula phrygia 14: +, 15: +, 16: +; Cardamine hirsuta 14: +, 22: +, 19: +; Carex divisa 8: 3; Carex divulsa ssp. divulsa 14: +, 22: +; Carex flacca ssp. serrulata 19: +; Centaurea salonitana 2: +, 3: +, 5: +; Centaurium erythraea ssp. rumelicum 13: +; Centaurium maritimum 16: +; Cerastium brachypetalum ssp. tenoreanum 21: +, 22: 1, 23: +; Cerastium brachypetalum ssp. roeseri 2: $+, 19:+$; Cerastium comatum 14: +, 12: +; Cerastium pumilum 4: 1, 9: +, 7: +; Glebionis segetum 16: +; Cichorium intybus 8: +, 14: +, 3: +; Cistus incanus ssp. incanus 2: +, 1: +; Cistus salvifolius 17: +; Clinopodium arvensis 2: +, 1: +; Clinopodium vulgare 21: +; Convolvulus arvensis 14: +; Crassula tillaea 6: +, 4: +, 7: +; Crupina vulgaris 3: +, 7: +; Cuscuta epithymum 20: +; Daucus broteri 2: +, 1: +, 7: +; Dianthus monadelphus ssp. pallens 3: +; Dianthus viscidus 19: +, 9: +; Dittrichia viscosa ssp. viscosa 13: +, 17: +; Draba muralis 14: +, 22: +, 18: +; Echinaria capitata 3: +; Echinops sphaerocephalus 2: +, 1: +; Ephedra major 14: +; Epilobium hirsutum 13: +; Erodium hoefftianum 13: +; Erophila verna ssp. macrocarpa 5: +; Erophila verna ssp. praecox 6: +; Erophila verna ssp. verna 8: 
Tab. 1. - continued

$+, 21:+$; Erysimum diffusum 23: +, 1: +; Euphorbia barrelieri ssp. thessala 21: +, 23: +, 9: +; Euphorbia chamaesyce 17: +; Euphorbia exigua 3: +, 18: +; Euphorbia falcata 20: +; Euphorbia myrsinites 2: +, 1: +; Euphorbia peplus 12: +, 16: +, 7: +; Euphorbia seguierana $\mathrm{ssp}$. niciciana 8: + , 23: +; Euphorbia taurinensis $2:+, 1:+;$ Festuca callieri 19: +; Festuca valesiaca $21:+, 19:+;$ Fumana thymifolia 18: +, 20: +; Gagea pusilla 11: +; Galium tenuissimum 8: +, 3: +; Galium tricornutum 11: +, 21: +; Gastridium ventricosum 13: +, 17: +; Genista carinalis 21: +; Geocaryum cynapioides 16: +; Geranium lucidum 14: +, 22: +; Geranium pusillum 2: +; Geranium robertianum 14: +, 19: +; Globularia alypum 18: +, 20: +; Gymnadenia conopsea 16: +; Hainardia cylindrica 3: +, 20: +; Herniaria glabra 21: +; Herniaria hirsuta 11: +, 22: +, 4: +; Herniaria hirsuta ssp. cinerea 5: +, 6: +; Hippocrepis ciliata 3: +, 20: +; Holcus annuus ssp. setiglumis 13: +; Hyparrhenia hirta 22: +, 9: +; Hypericum olympicum 21: +; Hypericum rumeliacum 21: +, 23: +, 3: +; Inula oculus-christi 23: +; Jasione heldreichii 15: +; Juncus capitatus 4: +; Kickxia elatine 16: +; Knautia species 3: +; Lagoecia cuminoides 14: +, 12: +; Lamium purpureum $8:+, 11:+$, Lathyrus cicera $2:+;$ Lathyrus sphaericus 19 : +; Legousia falcata 14: +, 3: +, 15: +; Lens nigricans 19: +; Leontodon saxatilis 4: 1; Limodorum abortivum 16: +; Linum nodiflorum 18: +; Filago minima 2: +, 4: +; Luzula forsteri 14: +, 21: +, 22: +; Lychnis coronaria 14: +; Matricaria chamomilla 10: +; Medicago arabica 8: 1, 11: +; Medicago coronata 14: +; Medicago falcata 14: +, 19: +; Medicago lupulina 1: +, 3: 1, 18: +; Medicago orbicularis 18: +, 19: +, 13: +; Melilotus neapolitanus $1:+;$ Mibora minima $4:+;$ Micromeria juliana 14: +; Microthlaspi perfoliatum 3: +; Micropyrum tenellum 8: +, 5: +; Minuartia glomerata ssp. macedonica 2: +, 1: +; Minuartia hybrida 3: +, 19: +, 4: +; Minuartia viscosa 8: +, 21: +; Moenchia mantica 14: 2, 19: +, 16: +; Myosotis incrassata 4: +, 7: +; Myosotis stricta 4: +; Neslia panicultata ssp. thracica 2: +, 1: +; Oenanthe silaifolia 14: +; Onobrychis aequidentata 3: 1; Ononis reclinata 20: +; Orchis species 13: +; Orlaya daucoides 19: +; Ornithogalum armeniacum 5: +; Paliurus spina-christi 14: +, 22: +; Pallenis spinosa 22: +, 20: +; Papaver rhoeas 10: +; Parvotrisetum myrianthum 22: +, 4: +; Petrorhagia illyrica $\mathrm{ssp}$ illyrica $23:+, 2:+$; Petrorhagia saxifraga $4:+, 9:+$; Phleum phleoides 10: +; Piptatherum coerulescens 18: +; Plantago afra 20: +; Pleurochaete squarrosa (only species in moose layer) 7: +; Poa angustifolia 11: +, 10: +; Poa pratensis 8: 1; Polygonum aviculare 4: +; Potentilla argentea 8: +, 11: +, 14: +; Potentilla pedata 8: +, 3: +, 10: +; Prunella laciniata 14: +; Ranunculus isthmicus 6: +; Ranunculus millefoliatus 8: +, 4: +, 7: +; Ranunculus muricatus 22: +; Ranunculus psilostachys $14:+$; Ranunculus rumelicus $8:+, 11:+, 5:+$; Raphanus raphanistrum 7: +; Rhagadiolus stellatus 3: +; Rorippa lippizensis 22: +; Rubia tinctorum 14: +; Salvia verbenaca 10: +; Scabiosa argentea ssp. ucranica $8:+, 23:+, 10:+$; Scabiosa sicula 18: +, 19: +; Scabiosa triniifolia 22: +; Prospero autumnale 2: +, 1: +, 4: +; Scolymus hispanicus 4: +; Podospermum canum 11: +, 3: +, 10: +; Securigera parviflora 12: +; Sedum amplexicaule 19: +; Sedum annuum 16: +; Senecio leucanthemifolius ssp. vernalis 8: +; Sideritis montana 18: +; Sideritis romana 5: +; Silene subconica 1: +; Silene cretica 19: +; Sisymbrium officinale 11: +, 5: +, 6: +; Sonchus arvensis 13: +; Spergula arvensis $11:+, 4:+$; Spergula pentandra $5:+, 6:+, 4:+$; Stachys angustifolia 19: +; Stachys cretica ssp. cassia $23:+;$ Stellaria pallida $14:+, 3:+;$ Stipa capensis $14:+$, 20: +; Stipa capillata 20: +; Taraxacum sect. Ruderalia 8: +, 5: +; Thymus odoratissimus 23: +, 10: +; Thymus sibthorpii 15: +, 4: +; Tolpis virgata $7:+$; Tordylium apulum 12: +; Tordylium maximum $3:+$; Torilis africana 19: +, 13: +; Tragopogon balcanicus 18: +; Tremastelma palaestinum $3:+, 18:+, 20$ : 1; Tribulus terrestris 4: +, 16: +, 7: +; Trifolium globosum 7: 1; Trifolium hirtum 19: +, 16: +; Trifolium pallidum 8: $+, 3: 1$; Trifolium repens $11: 1$; Trifolium grandiflorum 19: $+, 16:+;$ Trifolium vesiculosum 14: +; Medicago monspeliaca 18: +, 20: +, 5: +; Valantia muralis 14: +, 12: +; Valerianella dentata 2: $+, 1:+, 3:+$; Valerianella discoidea $3:+;$ Verbascum blattaria 21: $+, 22:+;$ Verbascum densiflorum 21 : +, 22: +; Vicia articulata 17: +; Vicia barbazitae 8: +; Vicia hirsuta 21: +; Vicia villosa 10: +, 17: +, 16 : + ; Xeranthemum annuum 23: $+, 1:+, 20:+$;

Localites of relevés, details of relevé are indicated in the following order: relevé number, country code, date (year/month/day), relevé area $\left(\mathrm{m}^{2}\right)$, altitude $(\mathrm{m})$, aspect (degrees), slope (degrees), cover herb layer (\%), description of locality, latitude, longitude. 1. GR, 20060428, 50, 340, 270, 1, 100, between Vrasna and Sohos, 40.71429, 23.62406; 2. GR, 20070513, 30, 426, 45, 1, 100, near to Askos, 40.75845, 23.41808; 3. GR, 20070513, 25, 162, 135, 2, 100, above Vrasna, 40.70946, 23.64963; 4. MK, 20070516, 30, 514, 225, 3, 95, Strumica, Novo Selo, 41.43231, 22.90296; 5. MK, 20070516, 30, 361, 180, 4, 100, Strumica, Novo selo, 41.42425, 22.89463; 6. MK, 20070516, 30, 571, 180, 5, 90 , Radoviš, Plačkovica, 41.65541, 22.47287; 7. MK, 20060424, 30, 247, 180, 8, 80, Nikolič, pasture in Q. coccifera zone, 41.26343, 22.73831; 8. MK, 20060424, 35, 265, 180, 15, 90, Nikolič, pasture in $Q$. coccifera zone, 41.26646, 22.73807; 9. GR, 20060426, 30, 225, 135, 5, 100, Kokinochori, $Q$. 
Tab. 1. - continued

coccifera zone, 40.82193, 24.02571; 10. GR, 20070515, 50, 320, 180, 2, 95, Chalkidiki, between Vavdos and Simantra, , 40.37739, 23.33592; 11. GR, 20070515, 30, 233, 225, 5, 95, Thesaloniki, Panorama, 40.57318, 23.03191; 12. GR, 20070515, 30, 316, 180, 1, 100, Chalkidiki, Vasilika between Agios Antonios and Monopigado, 40.43548, 23.11874; 13. GR, 20060407, 30, 390, 180, 2, 90, Thasos, above Prinos, 40.73500, 24.60870; 14.GR, 20060427, 40, 338, 180, 8, 90, Thasos, Megalos Prinos, 40.73328, 24.60799; 15. GR, 20070512, 30, 11, 180, 2, 90, Thasos, Aliki, 40.17827, 24.60262; 16. GR, 20070513, 30, 255, 180, 3, 100, Chalkidiki, between Olimpiada and Stratoni, 40.52979, 23.83467; 17. GR, 20060426, 40, 67, 135, 7, 90, Kokkinochoma (Kavala), 40.92415, 24.29526; 18. GR, 20070513, 30, 200, 225, 2, 90, Mirtofito, 40.78824, 24.21881; 19. GR, 20070513, 30, 210, 180, 2, 100, Askos, along the road to highway, 40.72011, 23.38634; 20, GR, 20070513, 30, 486, 180, 6, 100, Askos, 40.75688, 23.40912; 21. GR, 20070514, 30, 213, 180, 2, 100, Chalkidiki, between Porto Koufo and Kalamitsi, 39.97878, 23.96423; 22. GR, 20070514, 30, 52, 90, 2, 90, Chalkidiki, Skala Sikias, 40.02384, 23.99353; 23. GR, 20060428, 30, 41, 180, 4, 100, Kavala, Nea Peramos, 40.85714, 24.31423 .

its significance was assessed using the Monte Carlo permutation test with 999 permutations. The analysis was run with scaling for inter-sample distances to relate the gradient in the floristic composition to explanatory variables (TER BRAAK and ŠMILAUER 2002). Forward selection of explanatory variables was used to provide a ranking of the importance of specific variables and to avoid co-linearity (TER BRAAK and ŠMILAUER 2002). Variables with $\mathrm{p}=0.001$ were excluded from further analysis. We also calculated the total variance explained by individual variables and all variables together (LEPŠ and ŠMILAUER 2003) (Tab. 2 ). We estimated only the total explained variance, since it was impossible to make a model because, due to the high correlated climatic variable, the process would cause multi-co-linearity (SulLivan et al. 2011, AlahUTA et al. 2011). We then presented the most explanatory statistical variables in a RDA diagram (Fig. 5).

Tab. 2. Results of forward selection: significant environmental variables and percentage of the total variance of species data explained (\% TV), calculated with RDA.

\begin{tabular}{lccc}
\hline Variable & $\mathrm{P}$ & $\mathrm{F}$ & $\%$ TV \\
\hline Temperature seasonality & 0.001 & 2.75 & 11.6 \\
Precipitation seasonality & 0.001 & 2.75 & 11.6 \\
Temperature annual range & 0.001 & 2.72 & 11.5 \\
Precipitation of coldest quarter & 0.001 & 2.65 & 11.2 \\
Precipitation of driest month & 0.001 & 2.65 & 11.2 \\
Precipitation of driest quarter & 0.001 & 2.59 & 11 \\
Precipitation of wettest month & 0.001 & 2.55 & 10.8 \\
Min temperature of coldest month & 0.001 & 2.65 & 10.6 \\
Mean diurnal range & 0.001 & 2.44 & 10.4 \\
Precipitation of wettest quarter & 0.001 & 2.54 & 10.4 \\
Precipitation of warmest quarter & 0.001 & 2.32 & 9.9 \\
Mean temperature of coldest quarter & 0.001 & 2.25 & 9.7 \\
\hline Total & & & 60.1 \\
\hline
\end{tabular}




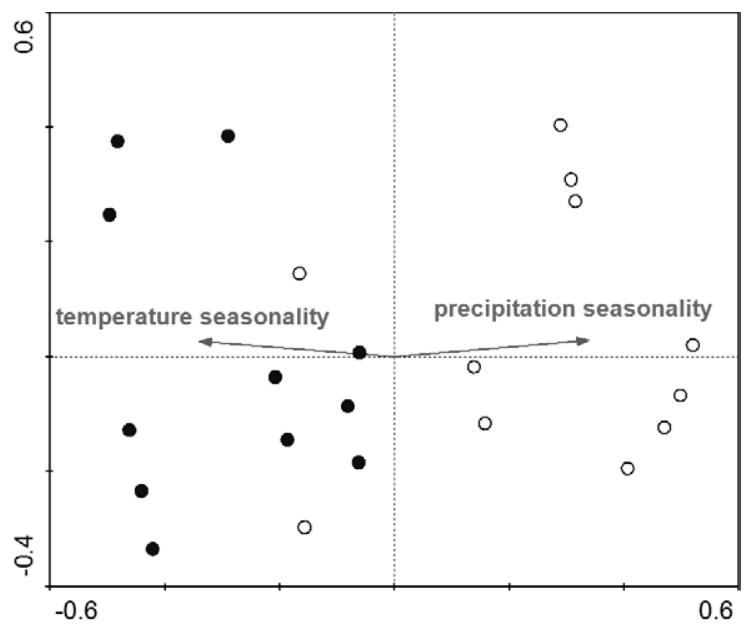

Fig. 5. Ordination diagram of sampling plots based on redundancy analysis (RDA). Only the two most important variables are included: seasonality in precipitation and temperature. Communities with Romulea bulbocodium are distributed in the interior part and represented by full circles; communities with Romulea linaresii ssp. graeca are distributed in coastal areas and represented by empty circles. First and second canonical axes have eigenvalues 0.117 and 0.050 .

We plotted the layers of seasonality of temperature and seasonality of precipitation on a geographical map of distibution of samples (relevés) in the southern Balkans (ELIÁš et al. 2013) to test the significance of results provided by previous analyses.

Life forms and the chorological spectra of groups were also determined following Raunkiaer 1934, Pignatti et al. 2005, Micevski (1993-2001), MicevsKi and Matevski (2005) and were passively projected onto the PCA diagram (Fig. 6).
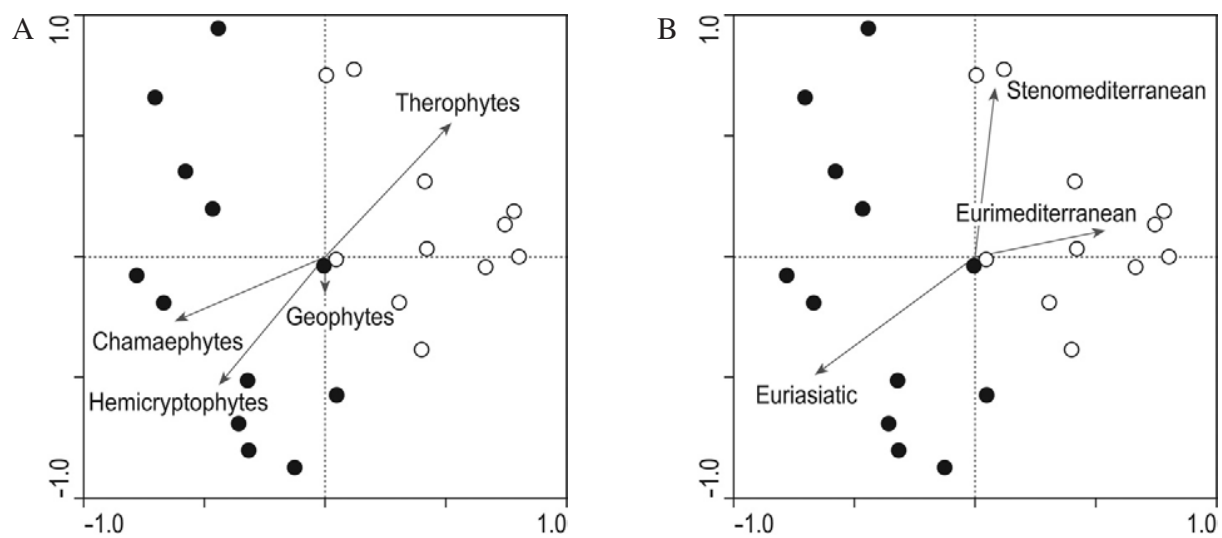

Fig. 6. Principal component analysis (PCA) of samples (relevés) with passive projection of life forms (A) and chorotypes (B). Communities with Romulea bulbocodium are represented by full circles and communities with Romulea linaresii ssp. graeca with empty circles. Eigenvalues of axes 1 and 2 are 0.279 and 0.189 . 


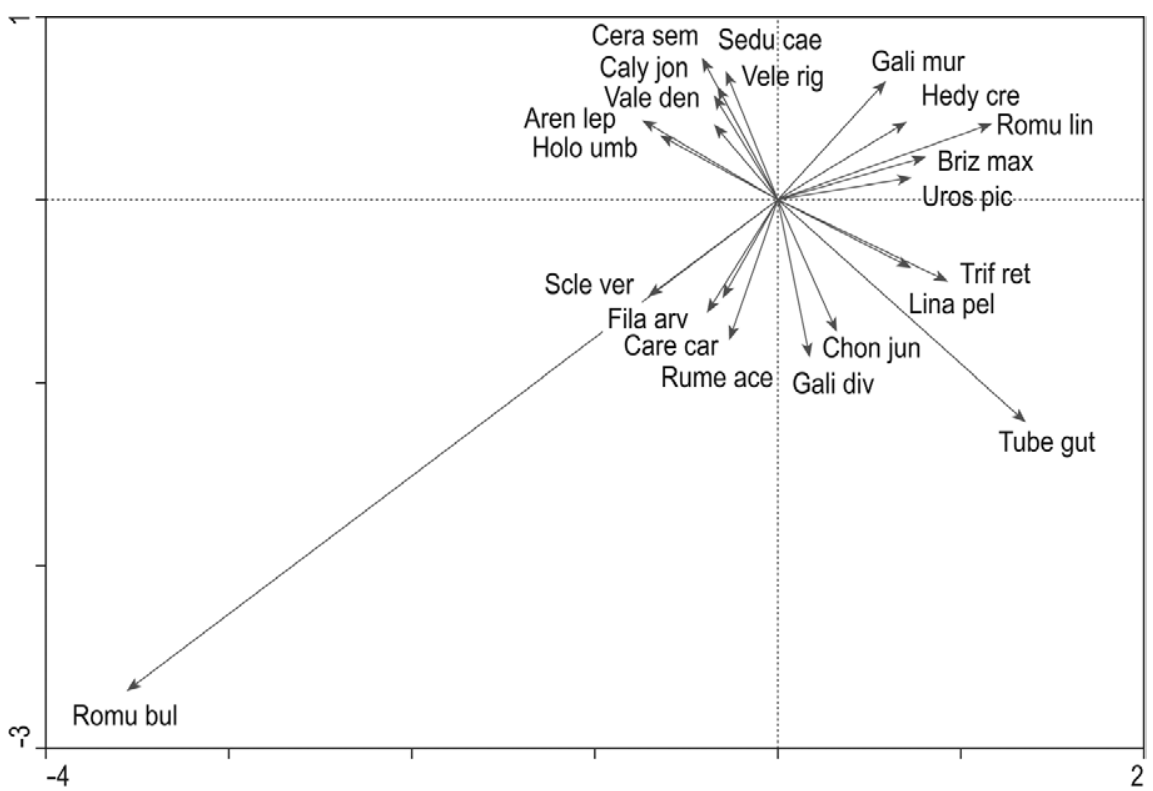

Fig. 7. PCA ordination of species. Fit for inclusion of species into ordination diagram was set to 45 . The species are presented by 4 letters for genus and 3 for species, for explanation compare table1.

The nomenclature of species follows the Euro+Med PlantBase (EuRO+MED 2006), except Amaranthaceae, Cistaceae, Dipsacaceae, Linaceae, Polygonacaea, Ranunculaceae, Rhamnaceae, Valerianaceae and Berteroa orbiculata following FLORA EUROPAEA and Medicago disciformis var. strumensis Velč. et Bond., Centaurium erythraea ssp. rumelicum (Velen.) Melderis and Pleurochaete squarrosa (Brid.) Lindb. Syntaxonomic nomeclature follows International Code of Phytosociological Nomenclature (ICPN) (WEBER et al. 2000).

\section{Results}

ESETG appear in areas under the influence of the Mediterranean climate (Fig. 3). Their northern limit corresponds to the distribution area of Quercus coccifera. ESETG appear in the coastal region of the Aegean Sea and in the interior along the rivers Struma and Strumica, where the influence of the Mediterranean climate can penetrate into the continent. ESETG do not appear in either agricultural areas or forests, only in areas with extensive grazing. They cannot therefore be found in the fertile plains along the Strymonas or Axios rivers, but appear generally on the edges of these areas, where grazing is still maintained.

The dendrogram (Fig. 4) shows two well defined groups of communities. The first represents ESETG in which Romulea bulbocodium appear. These communities thrive in the interior part of the area and are co-dominated by Carex divisa, Plantago lagopus, Poa bulbosa, Trifolium subterraneum and Tuberaria guttata. Romulea linaresii ssp. graeca appears in the other group of ESETG. These communities are found along the coast of the Aegean Sea and are co-dominated by Plantago lagopus, Poa bulbosa, Trifolium cherleri and Vulpia ciliata. 
Since the topological variables and management are fairly unique throughout the region, we tried to identify the main factor within all the climatic factors that causes the diversity of communities. We tested the significance of climatic data from WorldClim database. Since the significance of the following variables was low $(\mathrm{p}=0.001)$, they were excluded from analysis: annual precipitation, isothermality, annual mean temperature, mean temperature of warmest quarter, mean temperature of wettest quarter, max temperature of warmest month and mean temperature of driest quarter. These variables mainly sum up annual averages. The results show that the most important climatic feature that enables the diversity of ESETG in the region is pronounced changes in precipitation and temperature i.e. seasonality (Tab. 2). Higher precipitation seasonality can be found in the coastal regions, where there is pronounced summer hydric stress, which is less pronounced in the interior. Precipitation of the warmest quarter is higher in the interior, more precipitation can be found on the coast in the wettest period (i.e., autumn) (graph not presented in the text). Precipitation is more evenly distributed in the interior (Fig. 5). The situation is the opposite with temperature, with which seasonality is more pronounced in the interior, where lower temperatures are found during the winter. Among significant variables are the minimal and mean temperature of the coldest month and quarter, respectively. This shows that the diversity of ESETG is also caused by cooler winter temperatures (Tab. 2, Fig. 5).

Structural analysis showed that communities in the coastal area contain more therophytes (e.g., Briza maxima, Urospermum pycroides, Hedypnois rhagadioloides, Galium murale)(Fig. 6A). On the other hand, hemicryptophytes and chamaephytes are more abundant in the interior (Scleranthus verticillatus, Carex caryophyllea, Rumex acetosella).

In terms of chorological spectrum, there are more species with a Mediterranean distribution pattern (e.g., Galium murale, Hedypnois rhagadioloides, Velezia rigida) in the coastal communities, whereas Euroasiatic species are more common (Carex caryophyllea, Rumex acetosella, Filago arvensis) inland (Fig. 6B).

It was decided to assign the plant communities elaborated to two associations. Communities from the interior part of the region in which Romulea bulbocodium appears were classified as Lagopo-Poetum bulbosae and the other one, found in the coastal region, as Romuleo graecae-Poetum bulbosae. Diagnostic species as well as typification are given in the section nomenclature.

\section{Discussion}

Mediterranean vegetation is limited to the north, with a continental climate dominating the continental parts of the Balkans. The main climatic factor preventing penetration of Mediterranean vegetation into the interior of the Balkan Peninsula is winter frosts (ČARNI and Matevski 2005, Medail and Diadema 2009). At the same time, the Mediterranean climate, with summer droughts, is a strong ecological filter that does not allow continental flora to settle in the region (FILIBECK et al. 2012).

ESETG, often dominated by Poa bulbosa, can be found mainly on fresh fine clay soils on intensively grazed sites around settlements. Such vegetation is rarely found on carbonate bedrock, since erosion and degradation is faster (OBERDORFER 1954). It can be found on carbonate bedrock only in places (e.g., in the bottom of valleys) where colluvium offers a ref- 
uge for these communities. Garrigue dominated by Cistus creticus is often found around these stands (Č́ARNI et al. 2010).

The vegetation growth of these communities correlates with the annual precipitation regime. Germination of spring annuals and growth of most perennials begins soon after the first autumn rains. Growth is fairly slow during the winter months but vegetation is usually well established by February. Growth is fast in spring, with the peak of growth and seed set in May-June. By the end of June, most of the herbaceous vegetation is dry and the seed dispersed. Summer species, in this case C4 grasses, such as Achnatherum, Bothriochloa, Chrysopogon, grow during the summer and seed in September-October (PÉREZ-CAMACHO et al. 2012).

Analysis of climatic variables derived from WorldClim database shows that they are the most important variables for explaining the variance among ESETG $(60 \%)$ and are highly correlated (Tab. 3). It must be borne in mind that the Mediterranean climate provides a climatic envelope for ESETG on the large scale (GILINGHAM et al. 2012). On a smaller scale, the analysis showed that the most important factor for the variability of ESETG within the research area is seasonality. Variables that give information about average climatic conditions (mean temperature and precipitation) are of fairly low importance. Inter-annual changes in precipitation and temperature also appear to be responsible for variability in other grazed herbaceous communities (ZHANG et al. 2011, LOHMANN et al. 2012).

The RDA diagram shows that the highest seasonality of precipitation is within ESETG in which Romulea linaresii ssp. graeca appears and the lowest within that with Romulea bulbocodium. The situation is the reverse with temperature (Fig. 5).

Temperature seasonality (Fig. 3) is lowest on the coast of the Aegean Sea and at higher altitudes (ESETG do not appear there). On the other hand, precipitation seasonality is highest in the coastal area and diminishes gradually towards the continent (Fig. 3).

In the analysis of climatic variables, two outliers differing from the result of the classification (Fig. 4), can be found, relevés 19 and 20 in table 1. Both species of Romulea appear in these relevés. They were sampled above Lake Volvi (Chalkidiki, Greece) and the proximity of the lake probably influences the local climate.

The life forms spectrum shows the Mediterranean character of ESETG. In these communities, therophytic species form $65 \%$ of the floristic inventory of inland (Romulea bulbocodium) communities and $73 \%$ of coastal (Romulea linaresii) communities. Comparison with similar therophytic grasslands from Bulgaria (SopotLIEVA 2009) and the Republic of Macedonia (ĆUŠTEREVSKA et al. 2012) showed that there are $47 \%$ of therophytes in such grasslands in Bulgaria and 53-64\% in those of the Republic of Macedonia.

More therophytes can be found in the coastal communities, where summer drought is more pronounced. Summer drought is a limiting factor for perennials but they can extend their biological activity if water is available (PERÉz-CAMACHO et al. 2012). More evenly distributed precipitation gives an advantage to perennials in inland communities. More elements of perennial dry grasslands from the class Festuco-Brometea can be found in the inland communities (Tab. 1).

Analysis of chorotypes (geo-elements) showed that $66 \%$ of species with a Mediterranean (in the widest sense) distribution pattern appear in coastal communities and $59 \%$ in inland communities. Comparing these data with regions with more pronounced continentality 
(SOPOTLIEVA 2009, ĆuŠTEREVSKA et al. 2012), it can be seen that there are $41 \%$ of Mediterranean species in Bulgarian communities and 46-53\% in those of the Republic of Macedonia. This indicates that Mediterranean are quite different from continental communities. Relevés of such communities further to the south have not been published but similar therophytic communities of olive grove grassland show $65 \%$ of Mediterranean species (ČARNI and MATEVSKI 2005).

In a comparison with the synthetic table made by OBERDORFER (1954) in the same region, many differences in the floristic composition of ESTEG can be found. However, these differences are very difficult to evaluate since changes appear in the management regime (abandonment), climatic changes, sometimes even differences in taxonomical concepts. In the period 1950-1999, precipitation decreased and summer temperatures rose in the eastern Mediterranean (NASTOS et al. 2013) while land use change and abandonment of traditional agriculture is one of the crucial problems in the Mediterranean landscape (BAJOcCO et al. 2012).

\section{Nomenclature}

OBERDORFER (1954) classified ESETG within the order Helianthemetalia and class Thero-Brachypodietea. The class Thero-Brachypodietea is nowadays limited to Mediterranean perennial pseudosteppe communities (RoDwELL et al. 2002). The vegetation under consideration is classified within the class of Mediterranean terrestrial plant communities dominated by annual low-growing herbs and grasses Helianthemetea guttati $(\mathrm{Br}$-- $\mathrm{Bl}$. in Br.-Bl. et al. 1952) Rivas Goday et Rivas-Mart. 1963 and order of Mediterranean and sub-Mediterranean ephemeral communities on acid soils and fire-prone habitats Helianthemetalia guttatii Br.-Bl. in Br.-Bl. et al. 1940 (Rodwell et al. 2002, AlLEGREZZA et al. 2006, FANELLi et al. 2010, RiBeIRo et al. 2012).

It would be also possible to classify the alliance Romulion - within the class Poetea bulbosae and order Poetalia bulbosae, which is found in Mediterranean and sub-Mediterranean heavily grazed pastures, trampled and manured by sheep (RODwELLet al. 2002, FARRIS et al. 2010) known till now only from the western Mediterranean region (CANO et al. 2007). As there appear climatic and floristic differences, the possible occurrence of these syntaxa in the eastern Mediterranean region should be studied in the future. ESETG cannot be classified within the class of ephemeral vegetation with winter annuals on bare or disturbed salt-marsh mud and sand Saginetea maritimae, since no halophilous plant species appear (e.g., Tomaselli et al. 2011) and classification of the alliance Romulion in the list of alliances (RoDWELL et al. 2002) should be reconsidered.

ESETG should be classified within the alliance Romulion, as proposed by OBERDORFER (1954). However, at first glance, OBERDORFer (1954) mentions only Poa bulbosa (that means a typical subspecies and not subspecies timoleontis) in list 1 (»Liste $\mathrm{I} \ll)$ as the dominant species of »Lagopeto-Poetum timolentis« and »Tortileto-Poetum timoleontis«, which would cause an invalid publication of the associations and, consequently, the alliance (ICPN, art. 3f, 8). However, according to THEURILlAT (pers. com.), OBERDORFER (1954: 88) says about synthetic lists 1-3 (»synthetische Listen I-III«) »... Brachypodium ramosum oder B. phoenicoides treten vollkommen zurück und werden durch Poa bulbosa (div. ssp.) oder Stipa tortilis ersetzt.« It is true that in »List $\mathrm{I} \ll$ Poa bulbosa is indicated as a species but 
in the other two lists (»Liste II « and »Liste III«), it is »Poa bulbosa coll.«. We would therefore be inclined to consider that a printing error occurred in the first list and that here, too, it should be interpreted as »Poa bulbosa coll.« (including also the subspecies timoleontis) on the basis of what is said on page 88. In this sense, Oberdorfer would use the name of the aggregate species in the list (Poa bulbosa coll.) but, on the other hand, would use a more narrowly defined taxon in the association name (Poa bulbosa ssp. timoleontis). From the point of view of articles $3 \mathrm{f}$ and 43 , both association names can thus be considered formally to be validly published, and so also the alliance name Romulion Oberdorfer 1954.

The correction of the name »Romulion« to »Romulion graecae« by BoLòs et al. (1996) cannot be accepted. Names such as »Romulion« should stay without the addition of a specific name, since there is no rule in ICPN about the correction of these names, and recommendation 10C cannot be applied.

\section{Romulion Oberdofer 1954}

Lectotype: Lagopo-Poetum bulbosae Oberdorfer 1954 corr. Čarni et al. 2014 - lectotypus hoc loco. Diagnostic species (OBERDORFER 1954): Allium guttatum, Alyssum minutum, A. repens, Campanula ramosissima, Gagea reticulata, G. chrysantha, Hedypnois rhagadioloides, Hypochoeris cretensis, Lagoecia cuminioides, Linaria simplex, Lotus angustissimus, Ornithogallum collinum, Ornithogalum armeniacum, Picris pauciflora, Romulea bulbocodium, $R$. linaresii ssp. graeca, $R$. columnae, Sedum aetnense, Silene graeca and Ziziphora capitata. Ecological conditions: early spring ephemeral therophytic grasslands on deeper soils in the eastern Mediterranean area.

The critical point of consideration of the nomenclature is the doubtful appearance of Poa bulbosa ssp. timoleontis (i.e., Poa timoleontis) in communities in the research area. During the field survey carried out in the southern part of the Republic of Macedonia and around Thessaloniki in Greece, we could not confirm Poa bulbosa ssp. timoleontis as appearing in the communities under consideration; only Poa bulbosa s. str. was identified. Since the area of research and also the species composition match Oberdorfer's »Lagopeto-Poetum timoleontis «, it was decided to correct the name of »Lagopeto-Poetum timoleontis « to Lagopo-Poetum bulbosae, on the basis of art. 43 of ICPN. We have not corrected the name of »Toriletum-Poetum timoleontis« appearing further to the south, since Poa timoleontis may appear there.

Lagopo-Poetum bulbosae Oberdorfer 1954 corr. Čarni et al. 2014 nom. corr. hoc loco

Neotype: Tab. 1, rel. 5 - neotypus hoc loco. Diagnostic species: Achillea coarctata, Alyssum desertorum, Carex caryophyllea, Poa bulbosa, Romulea bulbocodium and Teucrium capitatum. Ecological conditions: early spring ephemeral grasslands in the regions with less pronounced summer hydric stress.

Oberdorfer described this community under the name »Lagopeto-Poetum timolentis« [recte: »Lagopeto-Poetum timoleontis«]. Since the genus Lagopus has been validly published, the name »Lagopeto-Poetum timoleontis« must be maintained according to art. 14, and corrected to Lagopo-Poetum timoleontis Oberdorfer 1954 according to art. 41. At the same time, we would like to add a comment to the name »Tortileto-Poetum timoleontis«. Since no genus »Tortilis« exists, this must mean Stipa tortilis (art. 14), and the name should be corrected with the genus Stipa, i.e., Stipo tortilis-Poetum timoleontis Oberdorfer 1954. 
We also describe a new association, as: Romuleo graecae-Poetum bulbosae ass. nova hoc loco Holotype: Tab. 1, rel. 15 - holotypus hoc loco. Diagnostic species: Crepis zacyntha, Galium murale, Hedypnois rhagadioloides, Hordeum murinum ssp. leporinum, Lagurus ovatus, Poa bulbosa, Romulea linaresii ssp. graeca, Urospermum pycroides. Ecological conditions: early spring ephemeral grasslands in the regions with more pronounced summer hydric stress.

\section{Conclusions}

ESETG are typical elements of Mediterranean landscapes that are maintained by traditional land use. Since these are among the most diverse habitats in the region, the class Heliathemetea and alliance Romulion for the eastern Mediterranean, respectively, therefore also deserve to be listed in syntaxonomic interpretation manuals of the Habitat Directive among habitats 6220 Pseudosteppe with grasses and annuals of the Thero-Brachypodietea (FARRIS et al. 2007, BIONDI et al. 2012).

\section{Acknowledgement}

We are grateful to Jean-Paul Theurillat for his nomenclature clarification. We thank Iztok Sajko for preparation of geographical maps and other figures. The authors acknowledge financial support from the state budget through the Slovenian Research Agency (P1-0236) and a bilateral Macedonian-Slovenian project (SLO-MK/06-07/3).

\section{References}

Alahuhta, J., Heino, J., Luoto, M., 2011: Climate change and the future distributions of aquatic macrophytes across boreal catchments. Journal of Biogeography 38, 383-393.

AllegrezzA, M., Biondi, E., Felici, S., 2006: A phytosociological analysis of the vegetation of the central Adriatic sector of Italian peninsula. Hacquetia 5, 135-175.

Bajocco, S., De Angelis, A., Perini, L., Ferrara, A. Salvati, L., 2012: The impact of land use/land cover changes on land degradation dynamics: a Mediterranean case study. Environnemental Management 49, 980-989.

BARBERO, M., QueZel, P., 1976: Les groupements forestiers de Grèce Centro-Méridionale. Ecologia Mediterranea 2, 3-86.

Bergmeier, E., Blockeel, T. Böhling, N., Fournaraki, C., Gotsiou, P., Jahn, R., LansDOWN, R., TURLAND, N., 2011: An inventory of the vascular plants and bryophytes of Gavdopoula island (S Aegean, Greece) and its phytogeographical significance. Willdenovia 41, 179-190.

Biondi, E., Burrascano, S., Casavecchia, S., Copiz, R., Selvico, R., Galdenzi, D., Gigante, D., Lasen, C., Spampinato, G., Cenanzoni, R., Zivkovic, L., Blasi, C., 2012: Diagnosis and syntaxonomic interpretation of Annex I Habitats (Dir 92/43/EEC) in Italy at the alliance level. Plant Sociology 49, 5-37.

Bohn, U., Neuhäusel, R., Gollub, G., Hetter, C., Neuhäuslová, Z., 2003: Map of Natural Vegetation of Europe. Scale 1:2.500.000. Landwirtschaftsverlag, Münster. 
Bolòs de, O., Maselles, R. M., Ninot, J. M., Vigo, J., 1996: A survey on the vegetation of Cephalonia (Ionian islands). Phytocoenologia 26, 81-123.

Bracchetti, L., Carotenuto, L., Catorci, A., 2012: Land-cover changes in a remote area of central Apennines (Italy) and management directions. Landscape and Urban Planning 104, 157-170.

Braun-Blanquet, J., 1964: Pflanzensoziologie. Grudzüge der Vegetationskunde. 3. Auflage. Springer Verlag, Wien.

Cano, E., Ladero, M., García-Fuentes, A., Pinto-Gomes, C. J., Cano-Ortiz, A., 2007: Estado actual de la classe Poetea bulbosae en la Península Ibérica. Phytosociologia 37, 645-661.

Catorci, A., Ottaviani, G., Kosić, I. V., Cesaretti, S., 2012: Effect of spatial and temporal patterns of stress and disturbance intensities in sub-Mediterranean grasslands. Plant Biosystems 146, 352-367.

Chytrý, M., TichÝ, L., Holt, J., BotTA-DukÁt, Z., 2002: Determination of diagnostic species with statistical fidelity measures. Journal of Vegetation Science 13, 79-90.

ČARNI, A., MATEVSKI, V., 2005: Comparison of short-lived ruderal vegetation of the inland and coastal regions in the southern part of the Balkan Peninsula. Fitosociologia 42, 97-107.

ČARni, A. MATEVSKI, V., ŠILC, U., 2010: Morphological, chorological and ecological plasticity of Cistus incanus in the southern Balkans. Plant Biosystems 144, 602-617.

Ćušterevska, R., Matevski, V., Kostadinovski, M., Čarni, A., 2012: Dry grasslands communities of Erysimo-Trifolietum in the North-eastern part of the Republic of Macedonia. Hacquetia 11, 91-111.

Eliáš, P., Sopotlieva D., Dítě, D. HájKová, P. Apostolova, I., SenKo, D., MelečKová, Z., HÁJEK, M., 2013: Vegetation diversity of salt-rich grasslands in Southeastern Europe. Applied Vegetation Science 16, 521-537.

Euro+Med (2006): Euro+Med PlantBase - the information resource for Euro-Mediterranean plant diversity. Retrieved February 28, 2013 from http://ww2.bgbm.org/EuroPlus $\mathrm{Med} /$.

Fanelli, G., Blanco, P. M., De Sanctis, M., Serafini Sauli, A., 2010: The alliance Trachynion distachyae Rivas-Martínez 1978 in central Italy. Annali di Botanica 0, 39-51.

FARris, E., SECCHI, Z., FILIGHEDDU, R., 2007: Carattcerizzazione fitosociologica dell' habitat prioritario 6220- Pascoli substeppici di graminacee e piante annue dei Thero-Brachypodietea: caso di studio delle Sardegna settentrionale. Fitosociologia 44, 271-278.

Farris, E., Filigheddu, R., Deiana, P., Farris, G. A., Garau, G., 2010: Short-term effects on sheep pastureland due to grazing abandonment in a Western Mediterranean ecosystem: A multidisciplinary approach. Journal of Nature Conservation 18, 258-267.

Filibeck, G., Cornelini, P., Petrella, P., 2012: Floristic analysis of s high-speed railway embankment in a Mediterranean landscape. Acta Botanica Croatica 71, 229-248.

Flora Europaea Database: Royal Botanic Garden Edinburgh. Retrieved February 28, 2013 from http://rbg-web2.rbge.org.uk/FE/fe.html.

FrignANI, F., IIRITI, G., 2011: The genus Romulea in Italy: taxonomy, ecology and intraspecific variation in relation to the flora of Western Mediterranean islands. Fitosociologia 48 (Suppl. 1), 13-20. 
Gilingham, P. K., Palmer, S. C. F., Huntley, B., Kunin, W. E. Chipperfield, J. D., Thomas, C. D., 2012: The relative importance of climate and habitat in determining the distribution of species at different scales: a case study with ground beetles in Great Britain. Ecography 33, 831-838.

GusSEV, C., 2011: Submediterranean pseudo-steppes with annual herbs. In: BISERKOV, V., Gussev, C., Popov, V., Hibaum, G., Roussakova, V., Pandurski, I., et al. (eds.), Red data book of the Republic of Bulgaria, 3, Natural habitats, 152-154. IBEI-BAS \& MOEV, Sofia.

Hijmans, R. J., Cameron, S. E. Parra, J. L., Jones, P. G., Jarvis, A., 2005: Very high resolution interpolated climate surfaces for global land areas. International Journal of Climatology 25, 1965-1978.

Horvat, I., Glavač, V., Ellenberg, H., 1974: Vegetation Südosteuropas. G. Fischer, Stuttgart.

JANIŠOVÁ, M., BARTHA, S., KIEHL, K., DEngler, J., 2011: Advances in the conservation of dry grasslands: Introduction to contributions from the seventh European Dry Grassland meeting. Plant Biosystems 145, 507-513.

Kaligarič, M., Culiberg, M., Kramberger, B., 2006: Recent vegetation history of the North Adriatic grasslands: Expansion and decay of an anthropogenic habitat. Folia Geobotanica 41, 241-258.

KaVgaci, A., ČArni, A., BaŞARAn, S., BAŞARAn, M. A., KoŠIr, P., MarinŠEK, A., ŠIllc, U., 2010: Long-term post-fire succession of Pinus brutia forest in east Mediterranean. International Journal of Wildland Fire 19, 599-605.

LEPŠ, J., Šmilauer, P., 2003: Multivariate analysis of ecological data using CANOCO. Cambridge University Press, Cambridge.

Lohmann, D., Tietjen, B., Blaum, N, Joubert, D. F., Jeltsch, F., 2012: Shifting thresholds and changing degradation patterns: climate change effects on the simulated long-term response of a semi-arid savanna to grazing. Journal of Applied Ecology 49, 814-823.

Maning, J., GoldBlatt, P., 2001: A synoptic review of Romulea (Iridaceae: Crocoideae) in sub-saharian Africa, the Arabian Peninsula and Socotra. Adansonia 23, 59-108.

MATEVSKI, V., ČARNI, A., 2003: Spring nitrophyllous forest edge vegetation in the southern part of the Balkan Peninsula. Hladnikia 15-16, 73-83.

Mccune, B., MefFord, M. J., 2011: PC-ORD. Multivariate analysis of ecological data. Version 6. MjM Software, Gleneden Beach, Oregon.

Medail, F., Diadema, K., 2009: Glacial refugia influence plant diversity patterns in the Mediterranean basin. Journal of Biogeography 36, 1333-1345.

MicEVsKi, K., 1970: Astragalo-Potentilletalia, a new order of dry grasslands in Macedonia (In Macedonian). Prilozi 2, 15-23.

MicEvski, K., 1977: Erysimo-Trifolietum Micev. ass. nov. in the vegetation of Macedonia (In Macedonian). Prilozi 9, 75-82.

MiCEVSKI, K., 1985-2001: Flora of the Republic of Macedonia 1 (1-5) (In Macedonian). Makedonska akademija na naukite i umetnostite, Skopje.

Micevski, K., Matevski, V., 2005: Flora of the Republic of Macedonia 1 (6) (In Macedonian). Makedonska akademija na naukite i umetnostite, Skopje. 
Nastos, P. T., Politi, N., Kapsomenakis, J., 2013: Spatial and temporal variability of aridity index in Greece. Atmospheric Research 119, 140-152.

Natcheva, R., Ivanova, D., 2011: Report 73. In: Vladimirov, V., Dane, F., Matevski, V., Stevanović, V., Tan, K.(eds.), New floristic records in the Balkans: 15. Phytologia Balcanica 17, 144.

Nikolić, T. (ed.), 2000: Flora Croatica. Index florae Croaticae, 3. Natura Croatica 9 (Suppl. 1), $1-324$.

OBERDORFER, E., 1947: Gliederung und Umgrenzung der Mittelmeervegetation auf der Balkanhalbinsel. Berichte über das Geobotanisches Forschungsinstitut Rübel 1947, 84-111.

OBERDORFER, E., 1954: Nordägäische Kraut- und Zwergstrauchfluren im Vergleich mit den entsprechenden Vegetationseinheiten des westlichen Mittelmeergebietes. Vegetatio 5, 88-96.

ÖZdemír, C., BARAn, P., AKyol, Y., 2007: Morfological and anatomical study on Romulea linaresii Parl. subsp. graeca Beg. (Iridacaeae). Journal of Science and Technology 1, 319-326.

Peréz-Camacho, L., Rebollo, S., Hernández-Santana, V., Garciá-SAlgado, J., PaVón-GARCíA, J., GÓMEZ-SAL, A., 2012: Plant functional trait responses to interannual rainfall variability, summer drought and seasonal grazing in Mediterranean herbaceous communities. Functional Ecology 26, 740-749.

Peruzzi, L., Iiriti, G., Frignani, F., 2011: Contribution to the kariological knowledge of Mediterranean Romulea species (Iridaceae). Folia Geobotanica 46, 87-94.

Petrova, A., Vladimirov, V., 2009: Red list of Bulgarian vascular flora. Phytologia Balcanica 15, 63-94.

Pignatti, S. (ed.), 1982: Flora d'Italia. Edagricole, Bologna.

Pignatti, S., Menegoni, P., Pietrosanti, S., 2005: Bioindicazione attraverso le piante vascolari. Valori di indicazione secondo Ellenberg per le piante della flora d'Italia. Braun-Blanquetia 39, 1-97.

Pyankov, V. I., Ziegler, H., Akhani, H., Deigele, C., Luttage, U., 2010: European plants with C4-photosynthesis: geographical and taxonomic distribution and relations to climatic parameters. Botanical Journal of Linnean Society 163, 283-304.

RAKAJ, M., 2011: Floristic and chorological records for monocots of Lake Shkodra. In: Dursun, S., Zuchetti, M., Vosniakos, F. K., Mankolli, H. (eds.), Proceedings of the International Conference on Ecosystems, Tirana, 65-69.

Ribeiro, S., LAdero, M., Espírito-SAnto, M. D., 2012: Floristic composition patterns of Mediterranean annual non-nitrophilous grasslands in Eastern Portugal. Plant Biosystems 146, 534-549.

RAUnKIAER, C., 1934: The life forms of plants and statistical plant geography. Charendon Press, Oxford.

Rodwell, J. R., Schaminee, J. H. J., Mucina, L., Pignatti, S., Dring, J., Moss, D., 2002: The diversity of European vegetation. An overview of phytosociological alliances and their relationship to EUNIS habitats. Report EC-LNV 2002/054, Wageningen. 
Sopotlieva, D., 2009: Poo bulbosae-Achilleetum pseudopectinatae: a new plant association. Phytologia Balcanica 15, 235-244.

Sopotlieva, D., Apostolova, I., 2007: The association Erysimo-Trifolietum Micev. 1977 in Bulgaria and some remarks on its mediteranean character. Hacquetia 6, 131-141.

STEŠEVIĆ, D., 2002: Taxonomical-ecological-phytogeographical characteristics of flora of hill Gorica in Podgorica. Natura Montenegrina 1, 15-39.

Sullivan, C. A., Bourke, D., Skeffington, M. S., Finn, J. A., Green, S., Kelly, S., Gormally, M. J., 2011. Modelling semi-natural habitat area on lowland farms in western Ireland. Biological Conservation 144, 1089-1099.

Ter BraAk, J. F. C., ŠMilauer, P., 2002: CANOCO Reference manual and CanoDraw for Windows, User's guide to Canoco for Windows: Software for Canonical Community Ordination (version 4.5). Microcomputer Power, Ithaca, NY.

Tzonev, R. T., Dimitrov, M. A., Roussakova, V. H., 2009: Syntaxa according to the Braun-Blanquet approach in Bulgaria. Phytologia Balcanica 15, 209-233.

TICHÝ, L., 2002: Juice, software for vegetation classification. Journal of Vegetation Science $13,451-453$.

Tomaselli, V., Di Pietro, R., Sciendello, S., 2011: Plant communities structure and composition in three wetlands in southern Apulia (Italy). Biologia 66, 1027-1043.

TÜRKMEN, N., DüNZENLI, A., 2011: Early post-fire changes in Pinus brutia forests (Amanos Mountains, Turkey). Acta Botanica Croatica 70, 9-21.

Vassilev, K., Pedashenko, H., Nikolov, S. C., Apostolova, I., Dengler, J., 2011: The effect of land abandonment on the vegetation of upland semi-natural grasslands in the Western Balkans MTS, Bulgaria. Plant Biosystems 145, 654-665.

Weber, H. E., Moravec, J., Theurillat, J. P., 2000: International Code of Phytosociological Nomenclature. Journal of Vegetation Science 11, 739-768.

Zhang, G., Xu, X., Zhou, C. Zhang, H., OUYANG, H., 2011: Responses of grassland vegetation to climatic variations on different temporal scales in Hulun Buir Grasslands in the past 30 years. Journal of Geographical Sciences 21, 634-650. 